\title{
Special Section on Synchronization in Nonlinear Science and Engineering
}

Synchronization is a ubiquitous phenomenon of coupled nonlinear oscillators, commonly found in physics, engineering, biology, and other diverse disciplines. It has a long research history back to Christiaan Huygens, who discovered synchronized motion of two pendulum clocks in 1673. It is very easy to observe synchronization in our daily life: e.g., metronomes, candle fires, pet-bottle oscillators, saltwater oscillators, and so on (See, for example, experimental movies at http://www.youtube.com/user/IkeguchiLab?feature=watch).

For the last few decades, significant development has been made from both theories and experiments on synchronization of coupled limit cycle oscillators as well as coupled chaotic oscillators. Applications have been also developed to communication technologies, controlling techniques, and data analysis. Combined with the idea from complex network theory, neuroscience, and systems biology, the research speed of synchronization has been even accelerated. This Special Section of NOLTA is primarily dedicated to the recent advanced development of basics and applications of synchronization in science and engineering. A number of qualified works is included, ranging from experimental study on synchronization of Huygens' system, analog circuits, and singing voice to applied study of synchronization in communication networks. One invited paper is devoted to comprehensive reviews on generalized synchronization of chaotic oscillators.

On behalf of the editorial committee of the special section, the guest editors would like to express their sincere thanks to all the authors for their excellent contributions. In particular, they are grateful to Prof. Dr. Ulrich Parlitz for contributing his distinguished review article. They would also like to thank the reviewers and the members of the guest editorial committee, especially Prof. Hiroo Sekiya of Chiba University and the editorial staffs of the NOLTA journal, for their supports on publishing this Special Section.

Tohru Ikeguchi and Isao Tokuda

Guest Editors

\section{Editorial Committee of the Special Section}

\section{Guest Editors:}

Tohru Ikeguchi (Saitama University) and Isao Tokuda (Ritsumeikan University)

\section{Advisory:}

Yoshifumi Nishio (Tokushima University)

\section{Guest Associate Editors:}

Hiroya Nakao (Tokyo Institute of Technology), Hiroshi Kori (Ochanomizu University), Jun-nosuke Teramae (Riken), Marco Thiel (University of Aberdeen), Ulrich Parlitz (Max Planck Institute), Miguel A.F. Sanjuán (University Rey Juan Carlos)

\section{Associate Editors of NOLTA, IEICE:}

Igor Belykh (Georgia State University), Takehiko Horita (Osaka Prefecture University), Keiji Konishi (Osaka Prefecture University), Hiroyuki Torikai (Osaka University), Atsushi Uchida (Saitama University), Chai Wah Wu (IBM T. J. Watson Research Center)

\section{Secretary of the Special Section:}

Hiroo Sekiya (Chiba University) 